\title{
Periodontal Outcomes in Liver Transplantation Individuals: A Systematic Review and Meta-analysis
}

\author{
Tuélita M. Galdino ${ }^{1}$, Carla S. Oliveira ${ }^{1}$, Allyson N. Moreira ${ }^{2}$, Francisco Ivison Rodrigues Limeira ${ }^{3}$, Cláudia S. \\ Magalhães $^{2}$, Lucas G. Abreu ${ }^{4}$
}

1) Universidade Federal de

Minas Gerais, Belo Horizonte, Minas Gerais;

2) Department of Restorative Dentistry. Faculty of Dentistry, Universidade Federal de Minas Gerais, Belo Horizonte, Minas Gerais;

3) Faculty of Dentistry President Antônio Carlos. Alfa Unipac Teófilo Otoni, Minas Gerais;

4) Department of Child and Adolescent Oral Health. Faculty of Dentistry, Universidade Federal de Minas Gerais, Belo Horizonte, Minas Gerais; Brazil

Address for correspondence: Tuélita Marques Galdino, DDS, MSc.

Department of Restorative Dentistry, School of Dentistry, Universidade Federal de Minas Gerais. Av. Antônio Carlos, 6627, sala 3.342, Pampulha, Belo Horizonte, MG, Brazil. CEP: 31.270-901. tuelitamarques@yahoo.com.br ORCID: https://orcid. org/0000-0002-5522-7291

Received: 19.10.2020 Accepted: 14.01.2021

\begin{abstract}
Aim: To systematically review the literature for observational studies evaluating periodontal outcomes in pre- and post-liver transplant (LT) individuals compared to a control group.

Methods: Specific strategies were designed to search five databases and the grey literature. Hand-searches were also performed. The following periodontal outcomes were analyzed in pre-LT, post-LT and control groups: plaque index (PI), gingival index (GI), gingival growth (GO), alveolar bone loss (ABL) probing depth (PD) and clinical attachment level (CAL). Meta-analyses and subgroup analyses were conducted.

Results: Among the 257 studies retrieved, 11 studies were included. Four studies were incorporated into the meta-analysis and showed that PI (mean difference $=0.15,95 \%$ CI: $0.12-0.18$ ) and GI (mean difference $=0.31$, 95\%CI: 0.06-0.56) were significantly higher in post-LT individuals compared to the control group. There was no significant mean difference of PD (mean difference $=0.49$, 95\%CI: -0.26-1.25) but CAL (mean difference $=1.47,95 \%$ CI: $0.19-2.75$ ) were significantly higher in pre-LT individuals compared to the control group. Conclusion: The higher means of PI and GI in post-LT and CAL in pre-LT compared to healthy individuals suggest a relationship between the periodontal condition and the transplantation status.
\end{abstract}

Key words: periodontal disease - gingivitis - periodontitis - liver transplantation - oral health - systematic review.

Abbreviations: ABL: alveolar bone loss; ALT: alanine aminotransferase; AST: aspartate aminotransferase; BOP: bleeding on probing; CAL: clinical attachment level; CEJ: cemento-enamel junction; DIGO: drug-induced gingival growth; GO: gingival growth; GI: gingival index; GR: gingival recession; LT: liver transplantation; PD: probing depth; PI: plaque index.

\section{INTRODUCTION}

Periodontal health is the absence of clinically detectable inflammation in periodontal tissues. It is determined by the host response and its physiological conditions of achieving tissue homeostasis. There is a biological level of immune response that is consistent with clinical gingival health and homeostasis [1]. Periodontal diseases are complex multifactorial diseases involving the relationship among subgingival microbiota, immune and inflammatory response as well as environmental modifying factors.
Periodontal disease can be considered a modifiable risk factor for chronic liver disease [2]. Periodontitis can influence the progression of liver diseases, such as non-alcoholic fatty liver disease, cirrhosis and hepatocellular carcinoma, in addition to affecting liver transplantation (LT) [3-5]. Epidemiological studies have shown that individuals with periodontitis have an increase in important liver injury serum markers, such as aspartate aminotransferase (AST) and alanine aminotransferase (ALT) $[2,4]$.

Individuals after receiving a LT are predisposed to oral diseases due to permanent immunosuppression [6]. Furthermore, difficulty in oral hygiene, changes in saliva composition and in microbial flora can increase the pathogenic potential of dental biofilm [7]. Untreated oral diseases represent a potential risk of infection and sepsis, with an adverse impact on the health of organ transplant candidates or recipients [8, 9]. This impact may result in the postponement or cancellation of transplants or even in episodes of sepsis after the surgical procedure [8]. 
Thus, it is important to summarize the scientific evidence available for the periodontal condition of liver transplant patients. The aim of this study was to conduct a systematic review and meta-analysis of observational studies that investigated the periodontal condition in individuals undergoing liver transplantation. The null hypothesis was that the periodontal condition of LT individuals does not differ from patients without indication for LT.

\section{METHODS}

\section{Protocol and registration}

This systematic review and meta-analysis was registered on the International Prospective Register of Systematic Reviews (PROSPERO - University of York, York, UK) [10] (CRD 42018110594).

This study was reported in accordance with the guidelines of the Preferred Reporting Items for Systematic Reviews and Meta-Analyses (PRISMA) statement [11]. The investigated clinical question was as follows: Do individuals in pre- or postLT condition exhibit a more unfavorable periodontal status compared to patients without an indication for LT?

\section{Eligibility criteria}

Observational studies published in any language according to the PECOS question were included: Population $(\mathrm{P})$ : individuals of any age; Exposure (E): LT individuals; Comparison $(\mathrm{C})$ : patients without indication for LT (healthy); Outcome (O): any outcome related to periodontal condition; Study design (S): Case-control studies, cross-sectional studies or cohort studies.

Studies reporting the prevalence or incidence of periodontal disease in individuals undergoing LT without a control group, case report or series of cases report, literature review, qualitative studies and studies evaluating outcomes different from periodontal outcomes were excluded.

\section{Information sources}

Electronic searches were performed up to December 2017 and updated on December 2019 in five electronic databases: PubMed (http://www.pubmed.gov), Web of Science (http:// www.isiknowledge.com), Scopus (www.scopus.com), Ovid (http://gateway.ovid.com) and Lilacs (http://lilacs.bvsalud. org). The search strategy for PubMed was: (periodontal disease [MeSH] OR gingival disease [MeSH] OR gum disease $[\mathrm{MeSH}]$ OR periodontitis [MeSH] OR gingivitis [MeSH] OR pyorrhea) AND (liver transplantation [MeSH] OR hepatic transplantation [MeSH] OR liver transplant [MeSH] OR hepatic transplant [MeSH] OR liver grafting [MeSH] OR hepatic grafting). Search strategies specific to the other databases were tailored. No restrictions were imposed with regard to language or year of publication. The reference lists of the included articles and any literature review retrieved were hand-searched to identify studies that might have been missed in the electronic searches. Additionally, a partial grey literature search was conducted in Google Scholar limiting the search to the first 100 most relevant hits. The EndNote Web ${ }^{\oplus}$ (www.myendnoteweb.com) was used to organize the list of studies. Duplicate results were removed upon identification.

\section{Selection of studies}

Initially, two researchers (T.M.G. and C.S.O.) underwent a calibration process for the selection of studies by applying the inclusion and exclusion criteria. They discussed the criteria, which were applied to $10 \%$ of the references retrieved. This exercise was repeated until a satisfactory agreement was obtained based on Cohen's Kappa coefficient (coefficient=0.88). The study selection process was carried out in two phases. At Phase 1, the researchers (T.M.G. and C.S.O.) independently assessed the list with all titles/abstracts. The titles/abstracts that seemed to fulfill the eligibility criteria were selected for the Phase 2 of the selection process. At Phase 2, the same researchers (T.M.G. and C.S.O.) evaluated the full texts and applied the same eligibility criteria. Studies that met the eligibility criteria were included in this systematic review and meta-analysis. During the selection of studies, discrepancies between the two re-searchers were solved through discussion until a consensus was reached.

\section{Data extraction process and extracted items}

Two independent researchers (T.M.G. and C.S.O.) extracted the following data from the articles: name of the authors, year of publication, country where the study was conducted, study design, setting and LT phase information, sample size, age and gender of the evaluated individuals, measurement of the periodontal outcome evaluated, statistical analysis, and results of the comparison between groups. During data extraction, disagreements between the two researchers were solved through discussion until a consensus was reached. The authors of the included articles would be contacted if additional information was required.

\section{Assessment of risk of bias in individual studies}

Two researchers (T.M.G. and C.S.O.) independently assessed the risk of bias in the studies included using the Newcastle-Ottawa scale for cross-sectional studies. A quality score was calculated based on three main categories: adequate selection of groups (four items), comparability between groups (one item), and outcome and exposure assessment (three items). In the category appropriate selection of groups, the items evaluated were adequacy of case definition, representativeness of cases, adequacy of control selection, and definition of controls. In the category comparability between groups, the item that considers the number of confounders analyzed in the study was evaluated. In the outcome and exposure assessment category, we observed how exposure was determined, whether cases and controls were examined using the same method, and the non-response rate. Points were attributed to the items evaluated. Each item received a maximum of one point, except for the item in the group comparability category, for which a maximum of two points could be achieved. The final score could range from zero to nine points. The higher the score higher the methodological quality and lower the risk of bias. For the prospective study, the same categories were assessed: selection (representativeness of the exposed cohort, selection of the non-exposed cohort, ascertainment of exposure, demonstration that the outcome of interest was not present at start of study), comparability (number of confounders analyzed) and outcome (reliable 
assessment of outcome, follow-up long enough to outcome occur, adequacy of follow up or non-response rate). The score also varied from zero to nine points.

\section{Measures used}

This systematic review and meta-analysis considered outcome measures (means and standard deviations, and percentages) for comparison between LT individuals and healthy individuals.

\section{Data synthesis}

The studies included were assessed for methodological heterogeneity (characteristics of the subjects assessed and measurements of periodontal outcomes). Studies that presented methodological homogeneity were incorporated into a meta-analysis. The statistical heterogeneity $\left(\mathrm{I}^{2}\right)$ of the meta-analysis was also evaluated. For meta-analysis with an $\mathrm{I}^{2}$ greater than $40 \%$, the random effect model was used. For cases with an $\mathrm{I}^{2}$ lower than $40 \%$, the fixed effect model was used [12]. Continuous data (mean and standard deviation) were used in the meta-analysis.

\section{Subgroup analysis}

Depending on the periodontal outcome evaluated and the comparison between groups, subgroup analyses were performed. The results of subgroup analyses were given as difference in means and confidence interval (CI).

\section{Additional analysis}

If feasible, sensitivity analysis and publication bias assessment would be performed.

\section{RESULTS}

\section{Selection of studies}

The search in five databases retrieved 332 references. After removing duplicates, 257 titles/abstracts were evaluated at Phase 1. From these 257 titles/abstracts, 49 full texts were evaluated considering the eligibility criteria at Phase 2. Eleven studies that met the eligibility criteria were included in this systematic review and meta-analysis [6, 9, 13-21]. The 38 articles excluded after the evaluation of the full text and the reasons for exclusion are shown in the Supplementary File (Table I). Fig. 1 shows the flowchart of this systematic review and meta-analysis.

\section{Characteristics of included studies}

Of the eleven studies included, four were developed in Israel [13-17] one in Italy [18], one in the United States [9], one in Finland [6] one in Brazil [19], one in Germany [20], one in Kuwait [21] and one in the United Kingdom [14]. Ten included studies had a cross-sectional observational study design and one was a prospective controlled study design. All articles were published in English between 2000 and 2019. Among the eleven studies included, four evaluated pre-LT and post-LT groups

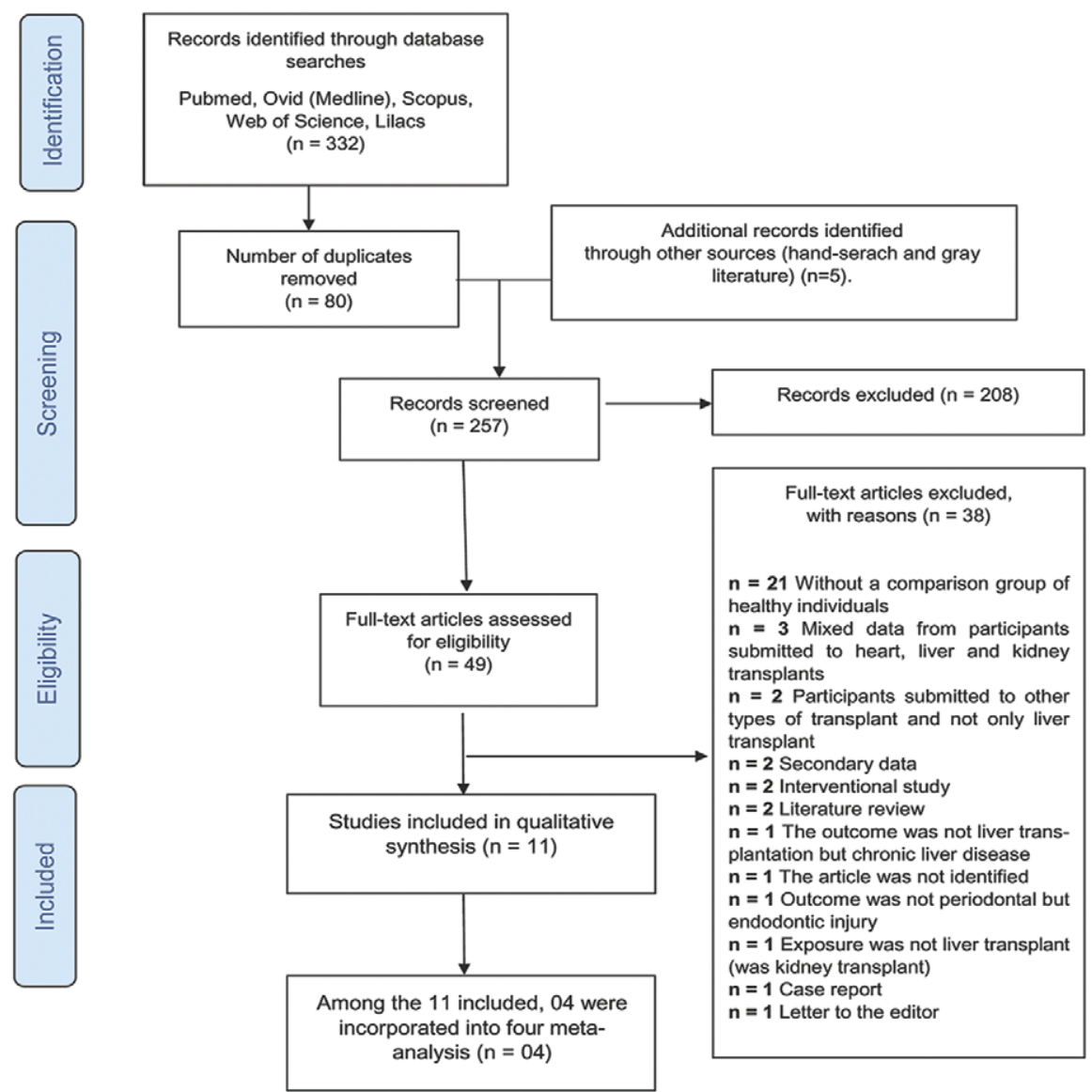

Fig. 1. Flowchart of the systematic review and meta-analysis. 
as well as a control group $[13,15,16,20]$, three evaluated a post-transplantation group and a control group $[6,17,18]$, two study evaluated a pre-transplantation group and a control group $[9,19]$, one study evaluated pre and post-transplantation participants in a single group compared to a control group [21] and one follow-up study evaluating individuals at baseline and at post-LT compared to a control group [14]. The studies were conducted in hospitals, medical institutes or dental clinics. Participants were children, adolescents, adults, and the elderly comprising both genders.

Periodontal outcomes were recorded using different indexes and panoramic x-rays analysis. Only two studies followed standardized criteria to define the case of periodontitis based on attachment loss $[19,20]$. In the study of OettingerBarak et al. [15], Ramfjord's plaque index (PI) [22], Loe's gingival index (GI) [23], PD: probing depth (PD), CAL: clinical attachment level (CAL), and gingival growth $(\mathrm{GO})$ were evaluated. Measurements of the vertical and horizontal increase of gingival tissue were made according to Seymour et al. [24]. Another study evaluated drug-induced gingival growth (DIGO) using the same index [6]. The study of Alanzi et al. [21] evaluated the GO using a different index developed by Aas 1963 [25] and modified by McGaw et al. 1987 [26] and PI was determined by the presence of visible plaque using the modified Quigley-Hein PI0. In two studies [17,18], the PI was classified as visible or not visible, according to Silness and Loe (1964), and gingival inflammation was classified by the presence or absence of bleeding 30 seconds after mild probing, according to Loe and Silness (1963). In a study [9], the frequencies of gingivitis and dental plaque and the number of teeth with mobility were described by the score indicating the presence or absence of the outcome. In one study, means (standard deviation) and medians (min-max) of PI, GI and GO index were recorded. The index used for PI was a modification of the index of O'Leary [14]. In one study, means of PD and CAL were assessed by individuals and mean percentage of sites with periodontal damage [19]. In two studies [13, 16], radiographic alveolar bone loss was characterized as the distance from the bone crest to the cemento-enamel junction (CEJ) measured by a software. The characteristics of the included articles are shown in the Supplementary File (Tables II-VI)

\section{Assessment of risk of bias in individual studies}

The results of the assessment of risk of bias in the included studies are presented in Supplementary File (Tables VII-VIII). All cross-sectional studies included presented adequate definition of the cases and clearly described the clinical examination used to collect data on the periodontal outcome. The adopted indexes and information on who performed the evaluations were recorded. Only one study [20] described who performed the evaluations and provided information on the employed classification of periodontitis (categories). In six articles, the samples consisted of representative cases with a correct description of periodontal indexes and conditions, setting of participants recruitment, and data collection period $[6,9,15,16,18,19]$. When one of these items was not clearly described, the requirement was considered incomplete due to a selection bias potential, which happened in four included articles $[13,17,20,21]$.
In eight included studies [13, 15-21], a correct definition of controls occurred. In five included studies [6, 15-18], confounding factors were controlled for the most important factor and for any additional factor during the analysis to compare the post-transplant group with the control group. In all included studies, an exposure assessment was reliably performed and an assessment of groups of exposed individuals was conducted in the same way as control groups. No response rate was reported in any of the included studies.

As regards the risk of bias of the prospective controlled study of Sheehy et al., [14], only two items were not fulfilled. For the representativeness of the exposed cohort for LT, the requirements were not satisfied in part, because the study did not consider two items of the domain selection of the Newcastle-Ottawa scale: the representativeness of the exposed cohort and the demonstration that outcome of interest was not present at the start of the study. Moreover, there was no demonstration that the outcome periodontal disease was not present at the study onset.

\section{Results of included studies \\ Studies with groups of pre-LT and post-LT individuals compared to a control group}

Oettinger-Barak et al. [15] analyzed PD, CAL, GO, Ramfjord's visible PI, and GI. There was no significant difference between the pre and post-transplant groups and the control as for PI and GI. GO, PD and CAL were greater for the pre- and post-LT groups compared to the control group. Barak et al. [13] and Oettinger-Barak et al. [16] evaluated a radiographic parameter by measuring the height of the alveolar bone crest from a reference point fixed to the JCE and found a greater bone loss in the pre and post-transplant groups compared to the control group (Supplementary File, Table II). Schmalz et al. [20] estimated the prevalence of healthy/ mild, moderate, or severe periodontitis with no significant differences among all three groups (pre-LT and post-LT individuals and a control group).

\section{Study with a group of pre-LT individuals compared to a control group}

The study of Guggenheimer et al. [9] included 300 individuals compared with a control group of individuals from organ procurement and transplantation network. Gingivitis, PI and the presence of two or more teeth with mobility were recorded. There was no difference between pre-transplant and control group regarding periodontal outcomes. Schmalz et al. [19] assessed 50 LT candidates and 50 individuals without liver disease who had undergone a complete periodontal examination. Gingival recession (GR), bleeding on probing (BOP), PI, PD, CAL and the number of missing teeth were recorded. Periodontitis was defined as $\geq 30 \%$ of teeth with proximal CAL $\geq 5 \mathrm{~mm}$. Pre-LT individuals presented means of GR, CAL and number of missing teeth significantly higher than the matched control group (Supplementary File, Table III).

\section{Studies with a group of post-LT individuals compared to a control group}

Periodontal condition was assessed by clinical parameters such as gingival inflammation [18], GI [17], visible PI [17,18], 
and DIGO [6] (Supplementary File, Table IV). One study showed a higher GI in the post-transplant group compared to the control group [17], while the other study found no differences between the groups for any parameter studied [18]. DIGO was higher in the post-LT group compared with the controls $[6,15]$.

Study with a single group of pre-LT and post-LT individuals compared to a control group

The study of Alanzi et al. [21] investigated the periodontal condition by plaque scores (modified Quigley-Hein plaque index) and GO, index developed by Aas 1963 [25] and modified by McGaw et al. [26]. Children who were scheduled to undergo LT and a control group with healthy children matched for age and gender were assessed. A higher mean PI and a positive correlation for GO index were observed in children of the transplant group compared with the controls (Supplementary File, Table V).

A prospective controlled study with LT individuals compared to a control group

This was a follow-up study that in the first evaluation of the GO index post-transplantation was not measured because there was insufficient time to analyze gingival enlargement. The number of individuals undergoing LT and the number of individuals in the control group varied at different times of measurement. Thus, PI, gingivitis and GO index were divided into subgroups. There were no significant changes with respect to the mean PI (\%) and GI (\%) for either the primary or permanent teeth between the LT patients and for the control group at each examination. The findings were similar for the GO index (\%) for the primary or permanent teeth at baseline (Supplementary File, Table VI).

Periodontal outcomes were categorized between primary teeth and permanent teeth [14]. Comparisons with other studies were unfeasible due to the unavailability of information in this regard. In this article [14], at baseline PI, GI and $\mathrm{GO}$ were measured by means of percentages, unlike other studies, in which different forms of measures (simple means or percentages) were used. Results on the post-transplant stage could not be compared, because the evaluation was in two stages. Both in the first (3 days post-transplant) and in the second stage (106 days post-transplant), the outcomes of periodontal disease was measured by means of percentages. At baseline, the measurements were different from data of other studies.

\section{Summary of results and subgroup analyses}

Four subgroup analyses were performed using continuous data (mean and standard deviation). The first compared the visible PI in a group of individuals after LT and a group of control individuals. Two studies were incorporated into this analysis [15,17]. The mean PI was significantly higher in post-LT individuals compared to the control group (mean difference $=0.15,95 \% \mathrm{CI}$ : 0.12-0.18, $\mathrm{I}^{2}=0 \%$ ) (Fig. 2). Two studies were incorporated into the second subgroup analysis that compared the GI of post-LT individuals and a control group $[15,17]$. The mean GI was significantly higher in post-LT individuals compared to the control group (mean difference $=0.31,95 \% \mathrm{CI}=0.06-0.56$, $\mathrm{I}^{2}=99 \%$ ) (Fig. 3).

The third and fourth subgroup analyses compared respectively the $\mathrm{PD}$ and the CAL of pre-LT individuals and a control group. Two studies were incorporated into these analyses $[15,19]$. There was no significant difference in the mean PD between pre-LT and healthy individuals (mean difference $=0.49$, 95\%CI: $-0.26-1.25, \mathrm{I}^{2}=96 \%$ ) (Fig. 4). However, the mean CAL was significantly higher in pre-LT individuals compared to the control group (mean difference $=1.47,95 \% \mathrm{CI}$ : $0.19-2.75, \mathrm{I}^{2}=95 \%$ ) (Figure 5).

\section{Additional analyses}

A sensitivity analysis for subgroup analysis with a high $\mathrm{I}^{2}$ was not possible because only two studies were incorporated into this subgroup analysis. Publication bias was assessed by means of funnel plots. The funnel plots demonstrated no

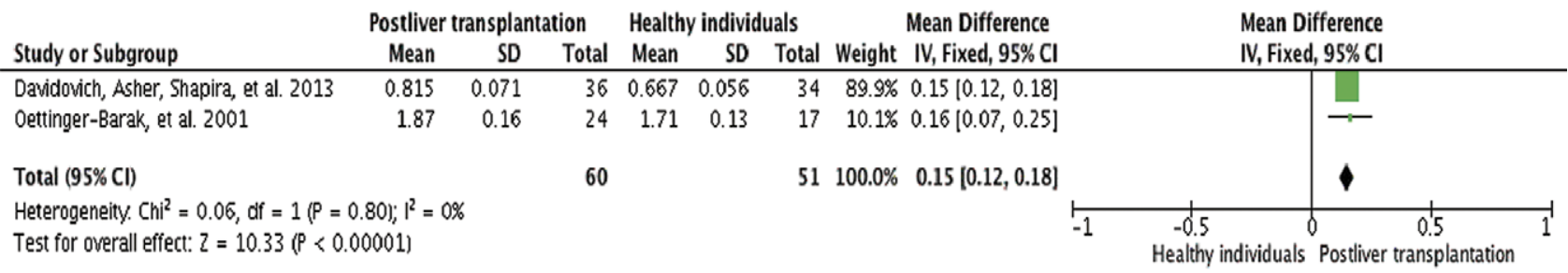

Fig. 2. Subgroup analysis of studies that analyzed the visible plaque index in a group of individuals after liver transplantation and a group of control individuals.

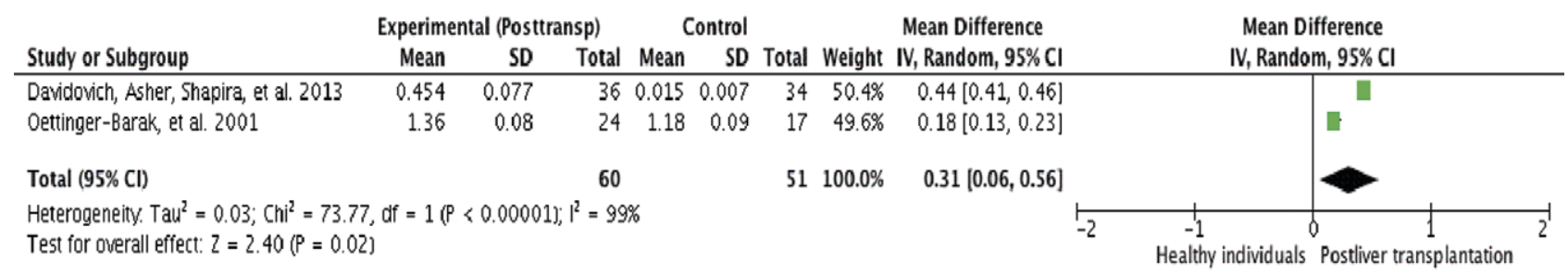

Fig. 3. Subgroup analysis of studies that analyzed the gingival index in individuals after liver transplantation compared to the control group. 


\begin{tabular}{|c|c|c|c|c|c|c|c|c|c|}
\hline \multirow[b]{2}{*}{ Study or Subgroup } & \multicolumn{3}{|c|}{ Pre liver transplantation } & \multicolumn{3}{|c|}{ Health individuals } & \multirow{2}{*}{\multicolumn{2}{|c|}{$\begin{array}{c}\text { Mean Difference } \\
\text { Weight IV, Random, } 95 \% \mathrm{Cl}\end{array}$}} & \multirow{2}{*}{$\begin{array}{l}\text { Mean Difference } \\
\text { IV, Random, } 95 \% \mathrm{Cl}\end{array}$} \\
\hline & Mean & SD & Total & Mean & SD & Total & & & \\
\hline Davidovich, Asher, Shapira, et al. 2013 & 2.7 & 0.7 & 50 & 2.6 & 0.6 & 50 & $49.2 \%$ & $0.10[-0.16,0.36]$ & -1 \\
\hline Dettinger-Barak, et al. 2001 & 3.32 & 0.24 & 13 & 2.45 & 0.23 & 17 & $50.8 \%$ & $0.87[0.70,1.04]$ & 달 \\
\hline Total $(95 \% \mathrm{Cl})$ & & & 63 & & & 67 & $100.0 \%$ & $0.49[-0.26,1.25]$ & \\
\hline $\begin{array}{l}\text { Heterogeneity } \text { Tau }^{2}=0.28 ; \text { Chi }^{2}=24 \\
\text { Test for overall effect: } Z=1.28(P=0.2\end{array}$ & $d f=1($ & 0.000 & $\left.1_{1}^{2}\right|^{2}=9$ & & & & & & $\begin{array}{c}-2 \\
\text { Healthy individuals }\end{array}$ \\
\hline
\end{tabular}

Fig. 4. Subgroup analysis of studies that analyzed the probing depth in individuals before liver transplantation compared to the control group.

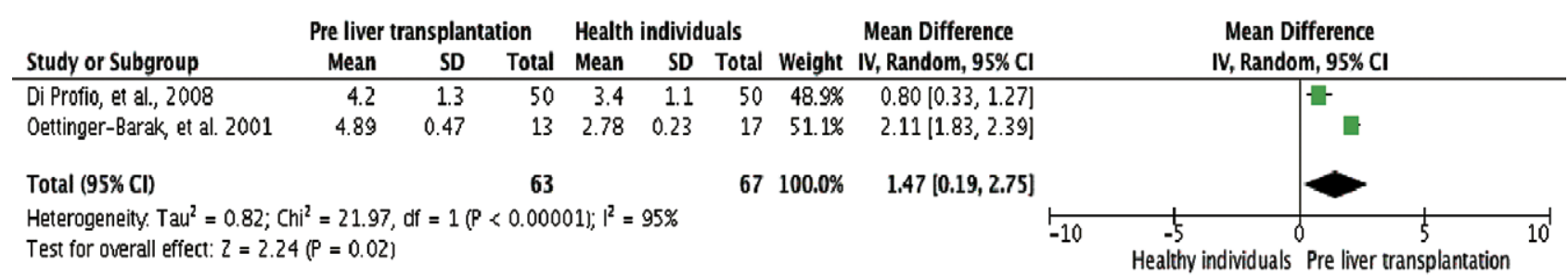

Fig. 5. Subgroup analysis of studies that analyzed the clinical attachment level in individuals before liver transplantation compared to the control group.

asymmetry (Fig. 6A-D). However, the analysis of publication bias was limited because only two studies were incorporated into each subgroup analysis.

\section{DISCUSSION}

Understanding the periodontal health-disease process is extremely important as periodontitis is a chronic disease that due to an intense inflammatory response triggers systemic changes [3]. Periodontal disease changes immunity and may result in infections (bacteremia) and respiratory and $[3,4]$ cardiovascular diseases [4], diabetes [4], and osteoporosis
[4]. In the present systematic review and meta-analysis, post-LT individuals had significantly higher means of PI, GI and alveolar bone loss compared to control individuals, suggesting an association between periodontal condition and LT situation.

The PI represents the level of subject oral hygiene, but it was used to assess periodontal condition in six studies $[14,15$, 17-19, 21] included in this review. The biofilm, which is one of the most important risk factors for gingivitis and periodontitis progression, may be supragingival or subgingival [27]. The various species of micro-organisms associated with periodontal diseases are highly metabolically specialized and organized
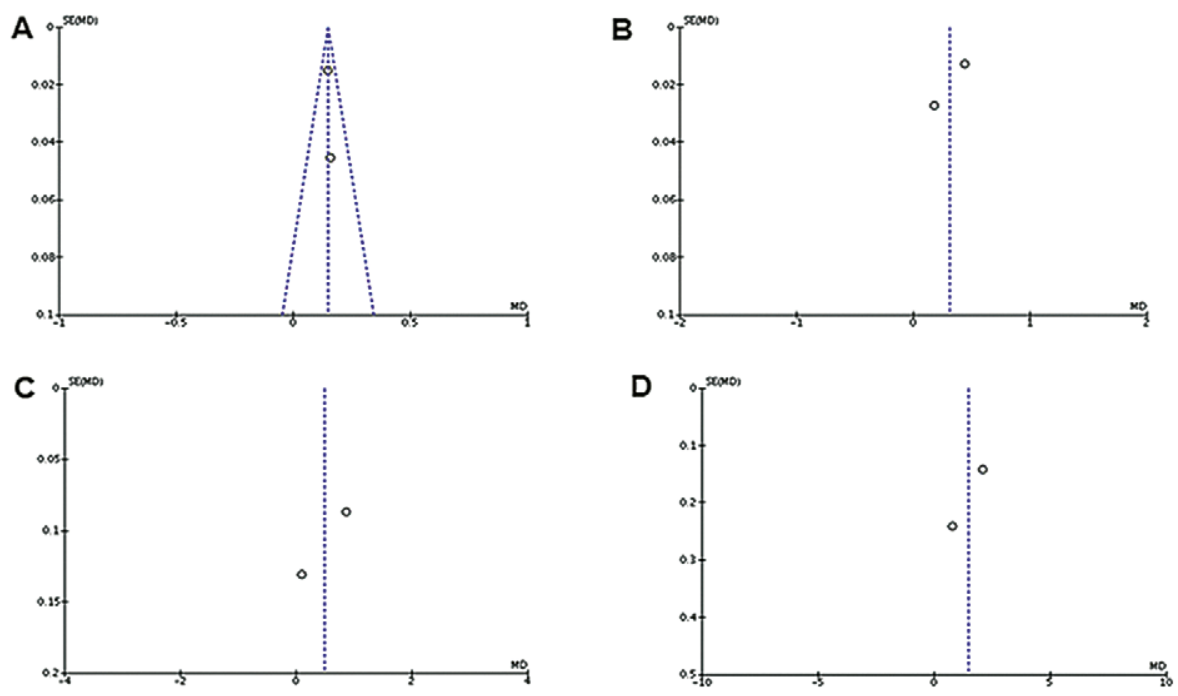

Fig. 6. Funnel plots. A) Subgroup analysis of studies that analyzed the visible plaque index in a group of individuals after liver transplantation (LT) and a group of control individuals. B) Subgroup analysis of studies that analyzed the gingival index in individuals after LT compared to the control group. C) Subgroup analysis of studies that analyzed the probing depth in individuals before LT compared to the control group. D) Subgroup analysis of studies that analyzed the clinical attachment level in individuals before LT. 
into multispecies biofilms. The proximity of microorganisms in this biofilm creates an opportunity for species to interact, leading to disease progression. These interactions can be synergistic or antagonistic and may produce a biofilm that prevents colonization by non-oral microorganisms. Some highly specialized microorganisms can subvert host defenses by releasing certain molecules, such as proteinases, leukotoxins and modified lipopolysaccharides (LPS), and disrupt the host immune response, which may result in the destruction of periodontal tissues in susceptible individuals [28].

After liver transplantation, individuals are submitted to a treatment with immunosuppressive medication [6] which is responsible for increasing the $\mathrm{pH}$ level in the oral cavity [17], making it more alkaline. At neutral $\mathrm{pH}$, saliva is supersaturated with calcium phosphate. When it becomes alkaline, the balance of the interaction of calcium and phosphorus ions is lost, and calcium phosphate precipitates, increasing the formation of dental calculus [29]. Saliva composition influences oral health and consequently disease activity [17]. About $48 \%$ of individuals with a pre-transplant chronic liver disease had a reduced salivary flow, which may influence the evolution of periodontal disease and other oral changes [30]. These individuals tend to suffer from problems such as $\mathrm{GO}$, gingivitis, dental hypoplasia and caries [17]. The main risk factor for stage I periodontitis is the partly calcified biofilm, that interacts with immune defenses of the host; this leads to gingival inflammation and eventually do deepening periodontal pockets [31]. Therefore, the abundant dental calculus found in LT recipients may probably reflect the increase in $\mathrm{pH}$ values and not in disturbances of salivary minerals [17].

Post-LT individuals had a significantly higher mean visible PI when compared to control individuals $[15,17]$, reflecting an inadequate oral hygiene $[17,18]$ which may lead to gingivitis. All pediatric patients who underwent or were scheduled to undergo LT had high plaque scores compared with healthy individuals [21]. Baygin et al. [32] also demonstrated an increase in the PI among children with a history of chronic liver disease. Similarly, Wondimu et al. [33] found increased $\mathrm{PI}$ in pediatric LT recipients. The mean of the GO in a single group of children pre-LT and post-LT was higher than among healthy controls [21]. Gingival growth was less prevalent among pediatric patients undergoing tacrolimus therapy [21].

The progression of oral health to the disease goes through a transient state of gingivitis, when inflammation and gingival bleeding are reversible if properly treated [34]. In gingivitis, the plaque buildup on the gingival margin leads to inflammation and a proportional increase in proteolytic bacteria and often anaerobic species [28]. A prolonged accumulation of biofilm may lead to inflammation in the supporting tissues and the formation of pockets harboring bacteria and serving as a focus of bacterial products and locally produced inflammatory mediators that can affect distant organs through the bloodstream [2]. Periodontitis appears to be associated with liver disease in the general population regardless of associated factors (alcohol use, smoking, meta-bolic risk, serum gammaglutamyltransferase levels, oral health care habits, lifestyle, and socioeconomic factors). As a preventable disease, periodontal disease may present a modifiable risk factor for chronic liver disease [2]. Therefore, the recommendations of oral health care providers for biofilm control and oral hygiene maintenance are necessary for transplant patients [35]. In addition to the conditions associated with the treatment of the liver condition, patients may have difficulties due to emotional stress, reduced monthly income, decreased ability, fear of death, dietary restrictions, changes in body image and practices of hygiene. Thus, psychological and educational social support may be necessary as part of the treatment [36]. Poor oral hygiene of transplant patients $[3,9,15]$ can be explained by the moment in which the patients' attention is focused on the other systemic diseases that currently affect the individual [37]. Oral health care may be neglected. Thus, it is recognized that post-transplant outcomes are influenced by social and behavioral determinants. The lack of oral hygiene and the need for periodontal treatment indicate a need for an early and comprehensive care for the prevention of diseases in transplant patients [21].

Individuals with chronic diseases do not have a regular preventive dental monitoring [17], which may lead to poor oral hygiene and gingivitis $[17,18]$. Deficiencies regarding oral hygiene stimulate gum growth [38]. The mean GI was significantly higher in the post-LT group compared to the control group [15, 17]. The prolonged immunosuppressive treatment after LT may also lead to several oral changes [17, 18], including GO, which is the most common adverse effect of cyclosporine therapy $[17,18]$.

Subgroup analyses showed that pre-LT individuals exhibited PD and CAL greater than controls subjects, but there was no significant difference for PD. Some factors may contribute to the explanation of this situation, such as their previous medical condition, for example in patients with liver cirrhosis [15], a lack of consistent oral hygiene, especially in the form of supplemental oral hygiene measures, such as the use of dental floss [39], as well as the high burden and the changes in lifestyles leading to a reduced priority for dental care [9].

Alveolar bone loss (ABL), as assessed by alveolar bone height based on digitized panoramic x-rays, was found to be greater in the pre and post-LT compared to the control group $[13,16]$. A meta-analysis for ABL could not be performed because the patients included in the studies of Barak et al. [13] and Oettinger-Barak et al. [16] were part of one and the same population, which has been studied by the same research group. Although liver function restoration occurs after transplantation, the bone loss is only partially reversible. Regardless of the preoperative bone density, most individuals undergoing LT will suffer bone loss in the first months after transplantation $[13,40]$. The bone loss is attributed to the effects of the transplant itself $[13,40]$, and also to the associated steroid therapy $[13,33]$. This contributes to the understanding that the mean radiographic ABL in pre- and post-LT individuals is significantly higher compared to control individuals since patients with liver damage undergo systemic inflammatory changes that may lead to attachment loss [41] and consequently to a greater ABL. After a severe bone loss during the first months following transplantation, restoration occurs and an increase in bone mass formation can be observed, reaching levels close to normal in two years [40]. The prevention of ABL will improve the periodontal condition of these patients, thus avoiding tooth loss associated with periodontal disease [13]. 
One of the difficulties found in the research related to periodontal outcomes is the wide variation in clinical measures used to assess the extent of periodontal changes and case definitions of periodontitis [3]. A diagnostic bias may occur in studies evaluating periodontal outcomes because they are based on response with different cutoff points. Only one included study evaluated both the parameters PD and CAL, which are fundamental for the definition of periodontitis cases. Therefore, it was not possible to estimate the overall risk of the disease. Thus, we considered the outcome measures common to studies evaluating the indexes and periodontal parameters in LT individuals compared with healthy individuals. These results are helpful to understand and try to explain the periodontal condition of patients undergoing pre- or post-LT and their possible systemic associations and implications in clinical practice.

Regarding the risk of bias assessment, seven studies had a score above five on the Newcastle-Ottawa scale (ranging from zero to nine points). All studies evaluated on this scale for this systematic review and meta-analysis presented an appropriate selection of transplant cases with a level of representativeness in most of them. Just one study [18] had an adequate control group selection: community controls. Most studies used convenience samples of individuals recruited in hospitals and dental schools. Convenience samples are easier and cheaper but may lead to estimates that may be unrepresentative of the given population [42]. In the comparison domain, eight studies adjusted the results for confounding variables related to lifestyle $[6,9,13,15-19]$. To control bias of two or more confounding factors regarding lifestyle, regression and/or correlation analyses were performed. The relationship between transplantation and periodontal indexes was adjusted for the variables habits of individuals. The proportional hazard was tested for the following covariates: presence of bleeding on probing, plaque and calculus [18], bone loss duration immunosuppressive therapy $[13,16]$, medications $[6,15]$, total protein concentration in saliva [17], current smoking [6, 9, 19], current use of alcohol [6] and time interval since LT [15]. In the exposure domain, the lack of information on nonresponse rates was the most common issue. No response rate was reported in any of the included studies. Individuals who left the study could be those with higher prevalence of periodontal indexes which might have biased the results. However, the examination of these individuals can be unfeasible due to time constrains or due to lack of patient cooperation because they were not well enough to cooperate for adequate examination [14].

In the risk of bias analysis of the prospective study of Sheehy et al. [14], only two requirements were not met. The representativeness of the exposed cohort did not satisfy the requirements in part for transplantation, even though data were collected among individuals from a defined catchment area. It was unclear whether the participants at baseline were free of periodontal disease. The existence of the disease at the start of the study could have overestimated the results. Studies with longer follow-up periods including comparisons of pre- and post-LT with a control group of healthy individuals provide important information on the impact of oral health care on the general health of patients.
This systematic review and meta-analysis primarily used data from cross-sectional observational studies. Thus, it is not possible to infer whether exposure to factors that led to periodontal conditions precede or are consequences of LT. Therefore, this design has a low efficacy to determine cause-effect associations, but identifies the individuals, the characteristics that can be managed, and the generation of causal hypotheses. Case-control studies investigating the etiology of periodontal conditions among LT patients and the determinants of longevity to assess health actions and services are recommended. Only one prospective cohort study was identified [14]. To avoid recall bias or case selection bias, we recommend the development of cohort studies with the follow-up of LT individuals, to verify the incidence of periodontal disorders and to investigate the factors to which these individuals are exposed to.

Based on the results of this systematic review and metaanalysis, there is a clear indication of the need of additional research on this topic. The available literature on the topic evaluated herein is unusual. Most existing studies have small samples, lacking methodological standardization for the diagnosis of periodontal conditions and evaluation of parameters and indexes that together lead to the descriptive recognition of periodontal disease.

\section{CONCLUSIONS}

Pre- and post-LT individuals have more adverse periodontal outcomes compared to healthy controls, such as: higher levels of plaque index, gingival index and radiographic alveolar bone loss in post-LT and higher levels of clinical attachment level in pre-LT individuals compared to healthy individuals. The majority of studies included had a cross-sectional design and their quality varied, highlighting the need for case-control and cohort studies to better elucidate the association of periodontal disease with liver transplantation and to determine cause and effect relations.

\section{Conflicts of interest: None to declare.}

Authors' contribution: T.M.G., A.N.M., F.I.R.L., C.S.M., L.G.A. conceived and designed the study. T.M.G., C.S.O., C.S.M., L.G.A. collected the data. T.M.G., C.S.O., F.I.R.L. analyzed the data. T.M.G., C.S.O., A.N.M., F.I.R.L., L.G.A. drafted the manuscript. T.M.G., A.N.M., C.S.M., L.G.A. critically revised the paper. All authors approved the final version to be published, and agree to be accountable for all aspects of the work.

Supplementary material: To access the supplementary material visit the online version of the J Gastrointestin Liver Dis at http://dx.doi. org/10.15403/jgld-3142

\section{REFERENCES}

1. Lang NP, Bartold PM. Periodontal health. J Periodontol 2018;89 Suppl 1:S9-S16. doi:10.1002/JPER.16-0517

2. Helenius-Hietala J, Suominen AL, Ruokonen H, et al. Periodontitis is associated with incident chronic liver disease-A population-based cohort study. Liver Int 2019;39:583-591. doi:10.1111/liv.13985 
3. Grønkjær LL. Periodontal disease and liver cirrhosis: A systematic review. SAGE Open Med 2015;3:2050312115601122. doi:10.1177/2050312115601122

4. Han P, Sun D, Yang J. Interaction between periodontitis and liver diseases. Biomed Rep 2016;5:267-276. doi:10.3892/br.2016.718

5. Pessoa LS, Pereira-da Silva FR, Alves EH, et al. One or two ligatures inducing periodontitis are sufficient to cause fatty liver. Med Oral Patol Oral Cir Bucal 2018;23:e269-e276. doi:10.4317/medoral.22204

6. Helenius-Hietala J, Ruokonen H, Gronroos L, et al. Oral mucosal health in liver transplant recipients and controls. Liver Transpl 2014;20:72-80 doi:10.1002/lt.23778

7. Parisi E, Glick M. Immune suppression and considerations for dental care. Dent Clin North Am 2003;47:709-731. doi:10.1016/s00118532(03)00038-7

8. Guggenheimer J, Mayher D, Eghtesad B. A survey of dental care protocols among US organ transplant centers. Clin Transplant 2005;19:15-18. doi:10.1111/j.1399-0012.2005.00251.x

9. Guggenheimer J, Eghtesad B, Close JM, Shay C, Fung JJ. Dental health status of liver trans-plant candidates. Liver Transpl 2007;13:280-286. doi:10.1002/lt.21038

10. PROSPERO. International database of prospectively registered systematic reviews in health and social care. J Clin Epidemiol 2018;100:103-110. Available at: http://www.crd.york.ac.uk/PROSPERO/. Accessed July 2.

11. Moher D, Liberati A, Tetzlaff J, Altman DG; PRISMA Group. Preferred reporting items for systematic reviews and meta-analyses: The PRISMA statement. PLoS Med 2009;6:e1000097. doi:10.1371/journal. pmed. 1000097

12. Higgins JP, Thompson SG, Deeks JJ, Altman DG. Measuring inconsistency in meta-analyses. BMJ 2003;327:557-560. doi:10.1136/ bmj.327.7414.557

13. Barak S, Machtei EE, Oettinger-Barak O, et al. Alveolar bone height in patients after liver transplantation. Transplant Proc 2000;32:718-720. doi:10.1016/s0041-1345(00)00956-8

14. Sheehy EC, Roberts GJ, Beighton D, O’Brien G. Oral health in children undergoing liver transplantation. Int J Paediatr Dent 2000;10:109-119. doi:10.1046/j.1365-263x.2000.00179.x

15. Oettinger-Barak O, Barak S, Machtei EE, Ardekian L, Baruch Y, Peled M. Periodontal changes in liver cirrhosis and post-transplantation patients. I: Clinical findings. J Periodontol 2001;72:1236-1240. doi:10.1902/ jop.2000.72.9.1236

16. Oettinger-Barak O, Machtei EE, Barak S, Baruch Y, Ardekian L, Peled M. Periodontal changes in liver cirrhosis and post-transplantation patients. II: Radiographic findings. Periodontol 2002;73:313-316. doi:10.1902/ jop.2002.73.3.313

17. Davidovich E, Asher R, Shapira J, Brand HS, Veerman CI, Shapiro R. Mucosal, pH, dental findings, and sali-vary composition in pediatric liver transplant recipients. Transplantation 2013;96:102-107. doi:10.1097/TP.0b013e3182962c58

18. Ferrazzano GF, Sangianantoni G, Cantile T, Iorio R, Ingenito A. Oral health status in liver transplant Italian children. Eur J Paediatr Dent 2013;14:323-327.

19. Di Profio B, Inoue G, Marui VC, et al. Periodontal status of liver transplant can-didates and healthy controls. J Periodontol 2018;89:13831389. doi:10.1002/JPER.17-0710

20. Schmalz G, Wendorff H, Berisha L, et al. Association between the time after transplantation and different immunosuppressive medications with dental and periodontal treatment need in patients after solid organ transplantation. Transpl Infect Dis 2018;20:e12832. doi:10.1111/ tid. 12832
21. Alanzi A, Alkheder M, Qudeimat M. Oral health status of Kuwaiti children with a history of chronic liver disease. Med Princ Pract 2019;28:341-346. doi:10.1159/000499594

22. Ramfjord SP. Indices for prevalence and incidence of periodontal disease. J Periodontol 1959;30:51-59. doi:10.1902/jop.1959.30.1.51

23. Loe $H$. The gingival index, the plaque index and the retention index systems. J Periodontol 1967;38 Suppl:610-616. doi:10.1902/ jop.1967.38.6.610

24. Seymour RA, Smith DG, Turnbull DN. The effects of phenytoin and sodium valproate on the periodontal health of adult epileptic patients. J Clin Periodontol 1985;12:413-419. doi:10.1111/j.1600-051x.1985. tb01377.x

25. Aas E. Hyperplasia gingivae diphenylhydantoinea. A clinical, histological, and biochemical study. Acta Odontol Scand 1963;21 Suppl 34:1-142.

26. McGaw T, Lam S, Coates J. Cyclosporin-induced gingival overgrowth: correlation with dental plaque scores, gingivitis scores, and cyclosporin levels in serum and saliva. Oral Surg Oral Med Oral Pathol 1987;64:293297. doi:10.1016/0030-4220(87)90007-7

27. Teles R, Teles F, Frias-Lopez J, Paster B, Haffajee A. Lessons learned and unlearned in periodontal microbiology. Periodontol 2000 2013;62:95162. doi:10.1111/prd.12010

28. Sanz M, Beighton D, Curtis MA, et al. Role of microbial biofilms in the maintenance of oral health and in the development of dental caries and periodontal diseases. Consensus report of group 1 of the Joint EFP/ ORCA workshop on the boundaries between caries and periodontal disease. J Clin Periodontol 2017;44 Suppl 18:S5-S11. doi:10.1111/ jcpe. 12682

29. Davidovich E, Davidovits M, Peretz B, Shapira J, Aframian DJ. The correlation between dental calculus and disturbed mineral metabolism in paediatric patients with chronic kidney disease. Nephrol Dial Transplant 2009;24:2439-2445. doi:10.1093/ndt/gfp101

30. Lins L, Bittencourt PL, Evangelista MA, et al. Oral health profile of cirrhotic patients awaiting liver transplantation in the Brazilian Northeast. Transplant Proc 2011;43:1319-1321. doi:10.1016/j. transproceed.2011.03.063

31. Heikkinen AM, Räisänen IT, Tervahartiala T, Sorsa T. Cross-sectional analysis of risk factors for subclinical periodontitis;active matrix metalloproteinase- 8 as a potential indicator in initial periodontitis in adolescents. J Periodontol 2019;90:484-492. doi:10.1002/JPER.18-0450

32. Baygin O, Cakır M, Ucuncu N. Oral and dental health in children with chronic liver disease in the Turkey Northeast. Niger J Clin Pract 2017;20:1182-1188. doi:10.4103/1119-3077.183259

33. Wondimu B, Nemeth A, Modeer T. Oral health in liver transplant children adminis-tered cyclosporin A or tacrolimus. Int J Paediatr Dent 2001;11:424-429. doi:10.1046/j.0960-7439.2001.00305.x

34. Mwatha A, Olson M, Souza S, et al. Gingival health and plaque regrowth response following a four-week interdental hygiene intervention. J Clin Dent 2017;28:A36-A44.

35. Ziebolz D, Hraský V, Goralczyk A, Hornecker E, Obed A, Mausberg RF. Dental care and oral health in solid organ transplant recipients:a single center cross-sectional study and survey of German transplant centers. Transpl Int 2011;24:1179-1188. doi:10.1111/j.14322277.2011.01325.x

36. Galvão AAF, Silva EG, Santos WN. The difficulties found by patients with chronic renal failure to start the treatment. Sci Ext Mag 2019;2:181 189.

37. Borrell LN, Crawford ND. Socioeconomic position indicators and periodontitis:ex-amining the evidence. Periodontol 2000 2012;58:69-83. doi:10.1111/j.1600-0757.2011.00416.x 
38. Kaswan S, Patil S, Maheshwari S, Wadhawan R. Prevalence of oral lesions in kidney trans-plant patients:a single center experience. Saudi J Kidney Dis Transpl 2015;26:678-683. doi:10.4103/1319. 2442.160128

39. Kauffels A, Schmalz G, Kollmar O, et al. Oral findings and dental behaviour before and after liver transplantation - a single-centre crosssectional study. Int Dent J 2017;67:244-251. doi:10.1111/idj.12290
40. Meys E, Fontanges E, Fourcade N, Thomasson A, Pouyet M, Delmas PD. Bone loss after orthotopic liver transplantation. Am J Med 1994;97:445450. doi:10.1016/0002-9343(94)90324-7

41. Alani A, Seymour R. Systemic medication and the inflammatory cascade. Periodontol 2000 2014;64:198-210. doi:10.1111/j.1600-0757.2012.00454.x

42. Shorten A, Moorley C. Selecting the sample. Evid Based Nurs 2014;17:32-33. doi:10.1136/eb-2014-101747 